\title{
Epileptic patients with de novo STXBPI mutations: Key clinical features based on 24 cases
}

\author{
'Chloé Di Meglio, ${ }^{2}$ Gaetan Lesca, 'Nathalie Villeneuve, ${ }^{3,4,5}$ Caroline Lacoste, ${ }^{3,5}$ Affef Abidi, \\ ${ }^{3,4,5}$ Pierre Cacciagli, ${ }^{6}$ Cécilia Altuzarra, ${ }^{7,8}$ Agathe Roubertie, ${ }^{9}$ Alexandra Afenjar, \\ ${ }^{9}$ Florence Renaldo-Robin, ${ }^{10}$ Bertrand Isidor, ${ }^{11}$ Agnes Gautier, ${ }^{12}$ Marie Husson, ${ }^{13}$ Claude Cances, \\ ${ }^{14}$ Julia Metreau, ${ }^{15}$ Cécile Laroche, ${ }^{16}$ Mondher Chouchane, ${ }^{17}$ Dorothée Ville, \\ ${ }^{17}$ Stéphanie Marignier, ${ }^{17}$ Christelle Rougeot, ${ }^{18}$ Marine Lebrun, ${ }^{19}$ Anne de Saint Martin, \\ ${ }^{20}$ Alexandra Perez, ${ }^{21}$ Audrey Riquet, ${ }^{3,4,5}$ Catherine Badens, ${ }^{4}$ Chantal Missirian, ${ }^{3,4,5}$ Nicole Philip, \\ 'Brigitte Chabrol, ${ }^{3,5}$ *Laurent Villard, and ${ }^{1,3,5} *$ Mathieu Milh
}

\begin{abstract}
SUMMARY
Objective: Mutations in the syntaxin binding protein I gene (STXBPI) have been associated mostly with early onset epileptic encephalopathies (EOEEs) and Ohtahara syndrome, with a mutation detection rate of approximately $10 \%$, depending on the criteria of selection of patients. The aim of this study was to retrospectively describe clinical and electroencephalography (EEG) features associated with STXBPI-related epilepsies to orient molecular screening.

Methods: We screened STXBPI in a cohort of 284 patients with epilepsy associated with a developmental delay/intellectual disability and brain magnetic resonance imaging (MRI) without any obvious structural abnormality. We reported on patients with a mutation and a microdeletion involving STXBPI found using array comparative genomic hybridization (CGH).

Results: We found a mutation of STXBPI in 22 patients and included 2 additional patients with a deletion including STXBPI. In 22 of them, epilepsy onset was before 3 months of age. EEG at onset was abnormal in all patients, suppression-burst and multifocal abnormalities being the most common patterns. The rate of patients carrying a mutation ranged from $25 \%$ in Ohtahara syndrome to $<5 \%$ in patients with an epilepsy beginning after 3 months of age. Epilepsy improved over time for most patients, with an evolution to West syndrome in half. Patients had moderate to severe developmental delay with normal head growth. Cerebellar syndrome with ataxic gait and/or tremor was present in $60 \%$.
\end{abstract}


Significance: Our data confirm that STXBPI mutations are associated with neonatalinfantile epileptic encephalopathies. The initial key features highlighted in the cohort of early epileptic patients are motor seizures either focal or generalized, abnormal initial interictal EEG, and normal head growth. In addition, we constantly found an ongoing moderate to severe developmental delay with normal head growth. Patients often had ongoing ataxic gait with trembling gestures. Altogether these features should help the clinician to consider STXBPI molecular screening.

KEY WORDS: Ohtahara syndrome, Early onset epileptic encephalopathy, Genetics, Suppression-burst.

\section{Key Points}

- We describe 24 patients carrying a mutation of $S T X B P 1$, from a large cohort of 284 epileptic patients

- $S T X B P 1$ mutations are found in approximately $10 \%$ of patient with an early onset epileptic encephalopathy, with a rate of $25 \%$ in Ohtahara syndrome

- Half of the mutated patients evolve to West syndrome, but only one of 24 patient was diagnosed with West syndrome from the beginning of the epilepsy

- Epilepsy improves after the first year of life in most mutated patients

- All of them have moderate to severe developmental delay with normal head circumference

The first mutation in the syntaxin binding protein 1 gene (STXBP1) was described in 2008. ${ }^{1}$ This mutation was a de novo microdeletion identified using microarray technologies in a girl with early infantile epileptic encephalopathy (EIEE) with a suppression-burst pattern on initial electroencephalography (EEG). When this gene was examined in patients with a similar phenotype, 14 of 43 unrelated patients were found to carry an STXBP1 mutation. ${ }^{1,2}$ Clinical picture at onset was quite stereotyped: neonatal onset of epilepsy, motor seizures (clonic, tonic, and spasms) occurring in a stormy way, abnormal EEG showing a suppression-burst pattern, and normal findings on brain magnetic resonance imaging (MRI). The implication of STXBP1 mutations in Ohtahara syndrome (OS) has been confirmed since then (EIEE4, MIM612164) ${ }^{1-7}$ Longitudinal follow-up of patients showed that, in most cases, seizures disappeared or became rare, and EEG dramatically improved in the first year of life. During childhood, severe developmental disability was constant, associated with movements disorders (dyskinesia, stereotypies, and tremor), which could be sometimes misinterpreted as epileptic seizures. ${ }^{4,8}$

Besides this description, mutations in STXBPI were found in various other conditions: infantile spasms with hypsarrhythmia $^{3,9}$ (West syndrome), early onset epileptic encephalopathy (EOEE) without specific epileptic syndrome, ${ }^{10,11}$ and late-onset infantile spasms with partial respiratory chain complex IV deficiency. ${ }^{12}$ Truncating mutations have also been reported in a few patients with nonsyndromic intellectual disability without history of epilepsy. ${ }^{13}$ Recently, Carvill et al. ${ }^{14}$ described three patients with Dravet syndrome not mutated for SCN1A but with a de novo STXBP1 mutation. Overall, the proportion of mutated patients was highly variable, according to the clinical presentation: $\sim 10 \%$ in cases of EOEE without specific epileptic/EEG features, ${ }^{10} \sim 2 \%$ in cohorts of West syndrome, ${ }^{3}$ and $<1 \%$ in intellectual disability without epilepsy. ${ }^{10,13}$ Key clinical features are needed to warrant molecular investigation of STXBPI and to increase the odds of finding a pathogenic mutation. ${ }^{3,4}$

The aim of this study is to identify the main clinical features associated with STXBP1-related epileptic encephalopathies. For that purpose we have screened STXBP1 in a large cohort of 284 epileptic patients and studied 22 patients with $S T X B P 1$ mutations to extract common key clinical features compared with nonmutated cases. We also reported two additional cases in which we found a microdeletion including STXBPI using array comparative genomic hybridization $(\mathrm{CGH})$.

\section{Patients and Methods}

\section{Patient selection}

We received 284 requests from various pediatric neurology centers in France to screen the STXBP1 gene for mutations between November 2010 and March 2014. The DNAs were initially sent to two groups (Lyon, $\mathrm{n}=73$; and Marseille, $\mathrm{n}=211$ ). The clinical data of non-mutated patients could be retrieved for all the patients whose DNA was sent to Marseille, since a specific questionnaire was sent with the DNA. There were 44 of 211 patients with Ohtahara syndrome, 45 of 211 patients with West syndrome as initial symptom (onset before 3 months in 20 patients), 50 patients with nonspecific EOEE, 6 patients with early myoclonic epilepsy, and 66 patients with nonspecific epileptic features beginning after 3 months. Overall, in the Marseille 
group, epilepsy began before 3 months of age in 145 patients, between 3 and 12 months in 51 patients, and after 12 months for only 15 patients. All the patients underwent clinical diagnostic STXBP1 gene sequencing in this study. The study was approved by the local ethics committee (CPP Sud Méditerranée reference 1153). All blood samples were obtained after receiving informed consent. We included two additional patients in whom a microdeletion including STXBP1 was found using array $\mathrm{CGH}$ analysis. The first (patient 3) was first addressed for STXBPl testing (found to be negative using Sanger sequencing); the second (patient 20), had a microdeletion identified without any a priori on a specific abnormality. Most of the patients in the cohort have had an array CGH analysis, but we cannot be certain that it was the case for all the patients. Clinicians ensuring the patient's follow-up described the phenotype of each patient by studying retrospective clinical history and neurologic examination. We paid particular attention to clinical and paraclinical elements associated in the literature with STXBP1 mutation: classification in epileptic syndrome according to the International League Against Epilepsy (ILAE) classification ${ }^{15}$; refractoriness of epilepsy; existence of epileptic spasms and/or hypsarrhythmic pattern on EEG; history of status epilepticus, nonepileptic movements, and fever sensitivity; and developmental status. We also reported on other features easily obtained: evolution of head growth, cerebellar signs (ataxic gait and trembling) and initial brain magnetic resonance imaging (MRI). We paid specific attention to characterize the following:

(1) Age at seizure onset (before 1 week, before 1 month, or before 3 months),

(2) Type of seizure,

(3) Number of seizures according to the age group,

(4) Electroencephalographic aspects,

(5) Antiepileptic drug response, and

(6) Age at the last seizure.

\section{EEG}

Patients with active epilepsy had electrophysiologic follow-up at least once a year with awake and sleep tracings. EEG data were analyzed by a pediatric neurologist or a neurophysiologist.

\section{MRI}

A first brain MRI was performed in all patients in the first year of the disease, and most often a control MRI was performed a few years after. Because MRI studies were performed in different institutions and sometimes several years ago, the imaging sequences could be quite variable, but all of the examinations contained anatomic sequences $\left(\mathrm{T}_{1}\right.$ - and $\mathrm{T}_{2}$-weighted) in the three axes (axial, coronal, and sagittal). The MRI images were analyzed by experienced radiologists.

\section{Molecular analysis}

DNA was extracted from peripheral blood for all patients and their parents. The 20 exons of STXBPI were amplified using polymerase chain reaction (PCR) (primer sequences are available upon request) and sequenced according to standard procedures on a $3500 \mathrm{XL}$ or a $3130 \mathrm{XL}$ automated sequencer (Life Technologies, Carlsbad, CA, U.S.A.). All mutations were confirmed using a second sequencing run and compared with parental DNAs to determine inheritance patterns. Mutations are numbered according to the GeneBank reference sequence NM_003165.

Array CGH and quantitative PCR: array CGH was performed using genomic DNA isolated from blood lymphocytes by standard methods, using Agilent $4 \times 180 \mathrm{~K}$ arrays (Agilent Technologies, Santa Clara, CA, U.S.A.). Microarrays were hybridized, scanned, and analyzed according to the manufacturer's instructions using an Agilent microarray analysis platform. Regions showing abnormal dosage were compared with regions already known to represent nonpathogenic copy number variations $(\mathrm{CNVs})$ listed in the database of genomic variants (http://projects.tcag.ca/cgibin/variation/gbrowse/hg18). Deletions were confirmed using quantitative PCR.

\section{RESULTS}

\section{STXBP1 molecular analysis}

Twenty different mutations were identified in 22 patients (Table S1). Except for patients 14 and 15, who were twins, patients were unrelated. The mutations were confirmed to have occurred de novo, except for the four patients for whom parental DNA was not available $(14,15,18$, and 22). Eight mutations were nonsense; 10 were missense mutations. Two patients had a deletion altering the STXBPI locus detected using array CGH: patient 20 carried an intragenic deletion of STXBPl involving exons 2-9 $\operatorname{arr}[\mathrm{hg} 19]$ 9q34.11 $(130,384,864-130,428,601) \times 1$, and patient 3 carried a larger deletion involving two OMIM genes: STXBPI and LRSAMI $\operatorname{arr}[$ hg 19] 9q33.3q34.11(129,548,333-130,533,312)x 1 (Table 1). There were no obvious correlations between the mutation class and the type or severity of epilepsy. We found only one recurrent mutation, in two unrelated patients (patients 2 and 22).

\section{Epilepsy features of the patients carrying a mutation of STXBP1}

A mutation in the STXBP1 gene was identified in 22 patients (8\%) among the 284 requests received, and in 24 patients in total if we include the patients with a microdeletion of $S T X B P 1$ found using array CGH. The median duration of follow-up was 4.5 years (10 months to 18 years). Table 1 summarizes clinical data from the patients.

The first neurologic symptoms were epileptic seizures in 22 of 24 patients, in 8 of 22 during the first week, in the maternity unit, and in 14 of 2 ? durino the first month of life 


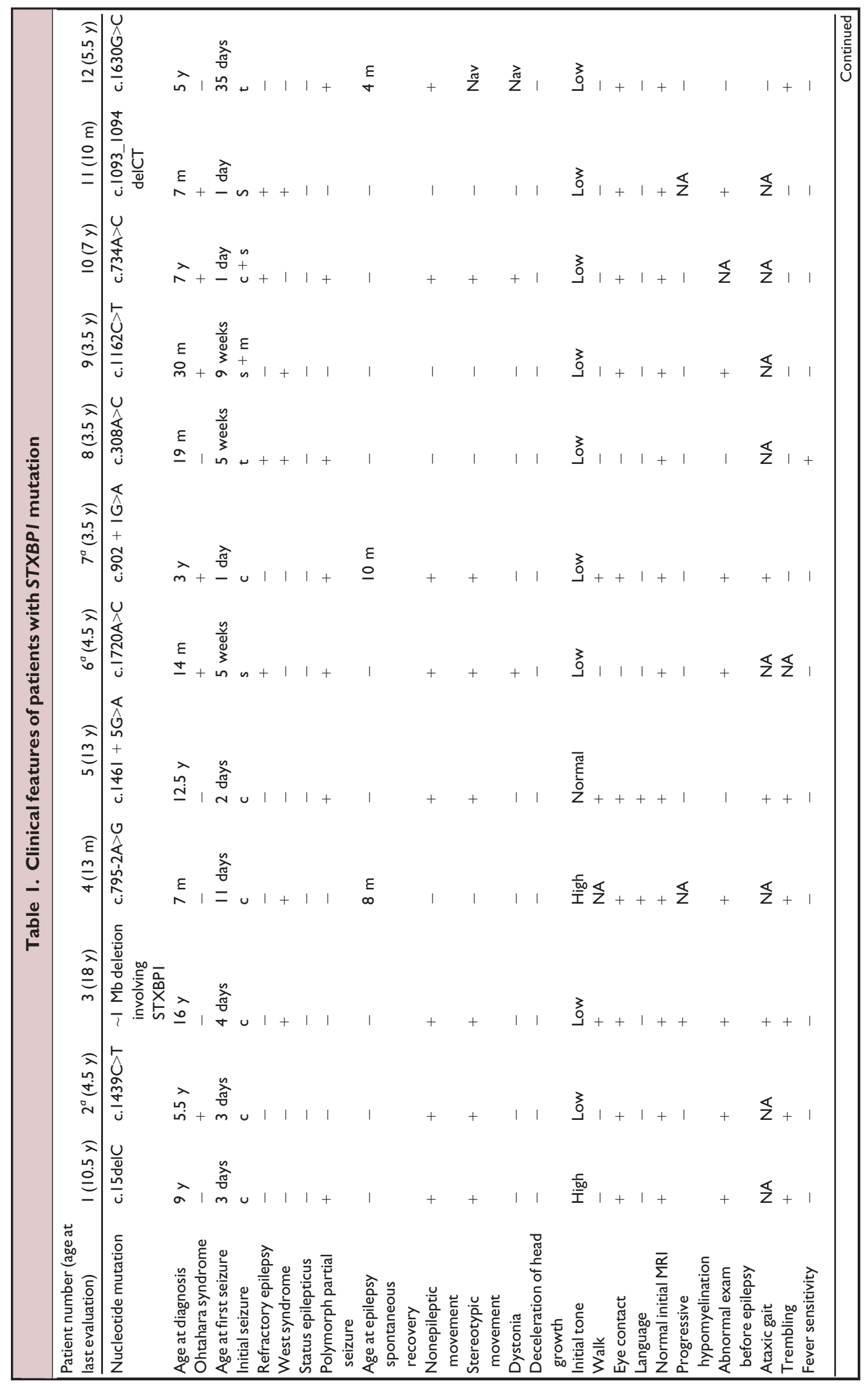




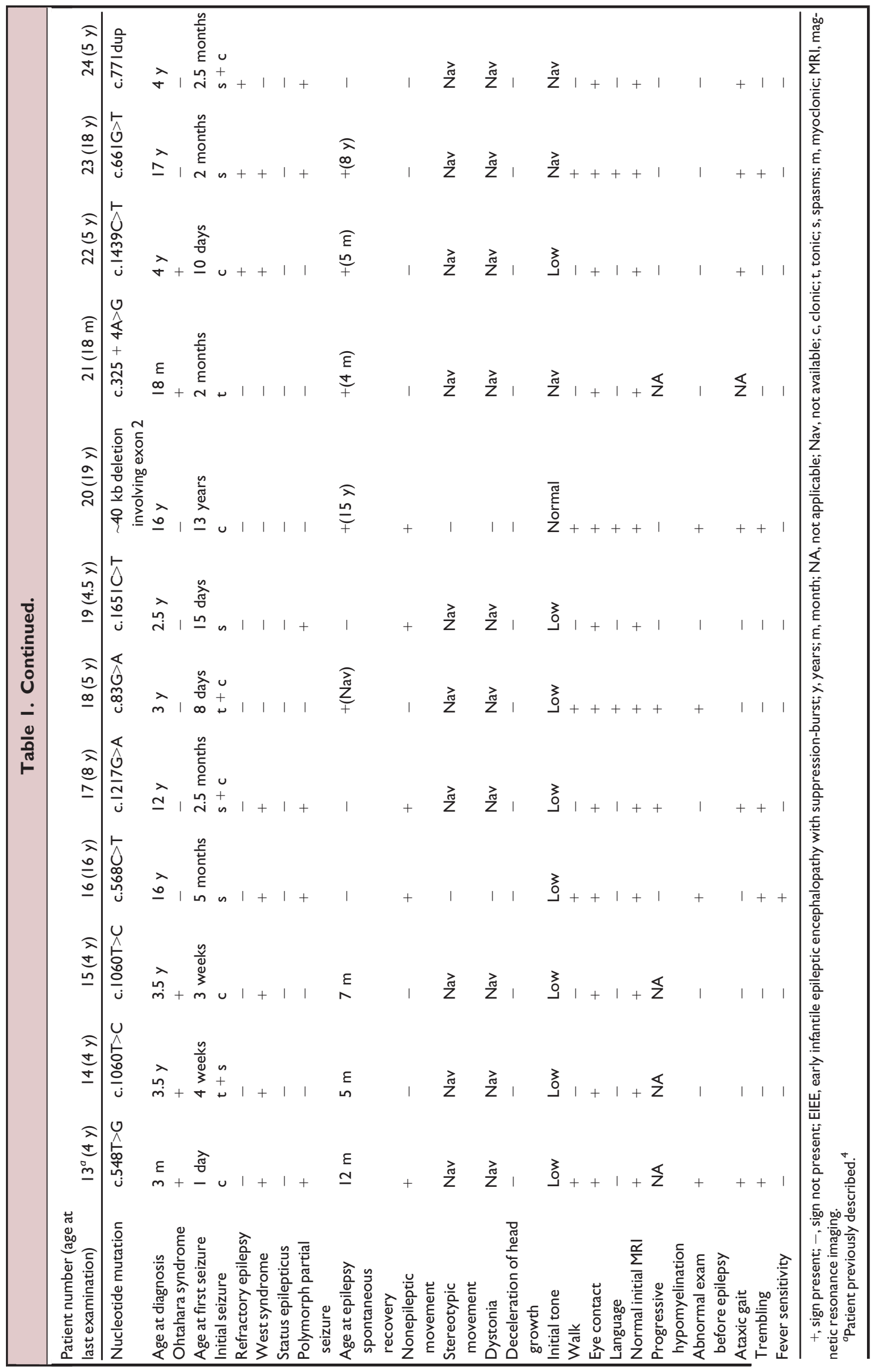


at home (Fig. 1). Two patients were already followed for a global developmental disorder when seizure occurred. One presented with epileptic spasms at 5 months, in the context of a severe developmental disorder. The second patient (patient 20) had ataxic gait and intellectual disability with seizure onset at 13 years (Table 1). Of interest, the molecular diagnosis was made "by chance" using array CGH analysis for this last patient.

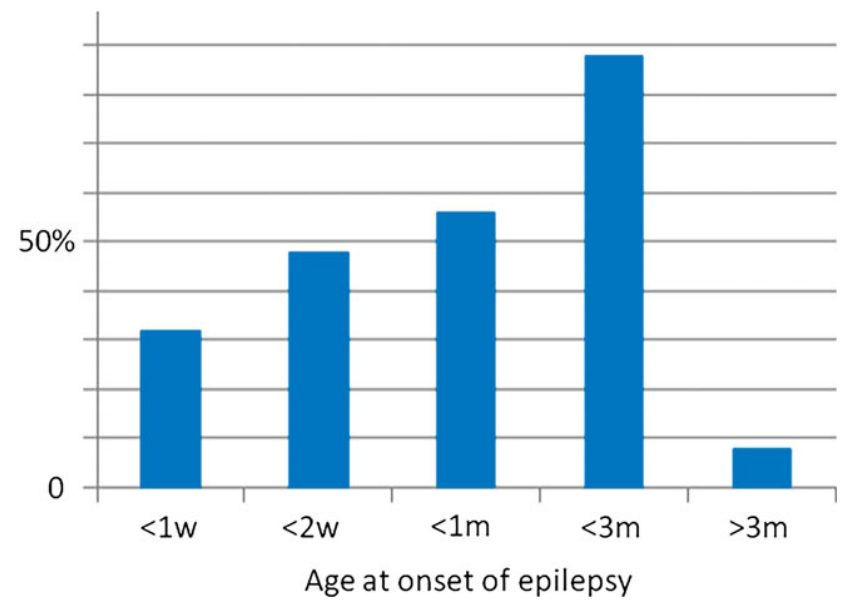

Figure I.

Cumulative distribution of mutated patients according to age of onset of epilepsy.

Epilepsia (c) ILAE
The first seizures were motor seizures in all patients. They were bilateral tonic asymmetric in three cases, clonic in 10 cases (unilateral in eight cases), isolated spasms in 8 cases, and bilateral axial myoclonic in 3 cases. Seizures were considered as bilateral or multifocal in 15 cases and focal in 9 cases. The first seizures occurred in a stormy way, but did not require an admission in intensive care unit.

Sensitivity to fever was noticed in two patients after the age of 6 months. We did not observe a specific response to any antiepileptic drug: we did not find any consistent efficacy of one antiepileptic drug, or any consistent worsening.

Among the 22 of 24 patients with an onset of epilepsy before 3 months of age, the initial EEG showed a suppression-burst pattern in 11 patients (50\%). Multifocal asynchronous spikes and slow waves without discontinuity were present in 6 of 22 (Fig. 2), and a hypsarrhythmic pattern was already seen from the first EEG in one at 2 months (patient 23). During the course of epilepsy, 10 of 22 patients presented West syndrome with epileptic spasms and hypsarrhythmia, and 3 of 22 patients had epileptic spasms without hypsarrhythmia. EEG normalized within the first year of life in 11 patients (including 5 patients with initial suppressionburst pattern). In the other cases, some focal spikes were observed. Surprisingly, the spikes were usually of high amplitude and resembled the so-called "functional" spikes usually seen in partial idiopathic epilepsies (see Fig. 1). Finally, three groups of patients could be delineated, based on the epileptic features within the first 2 years:
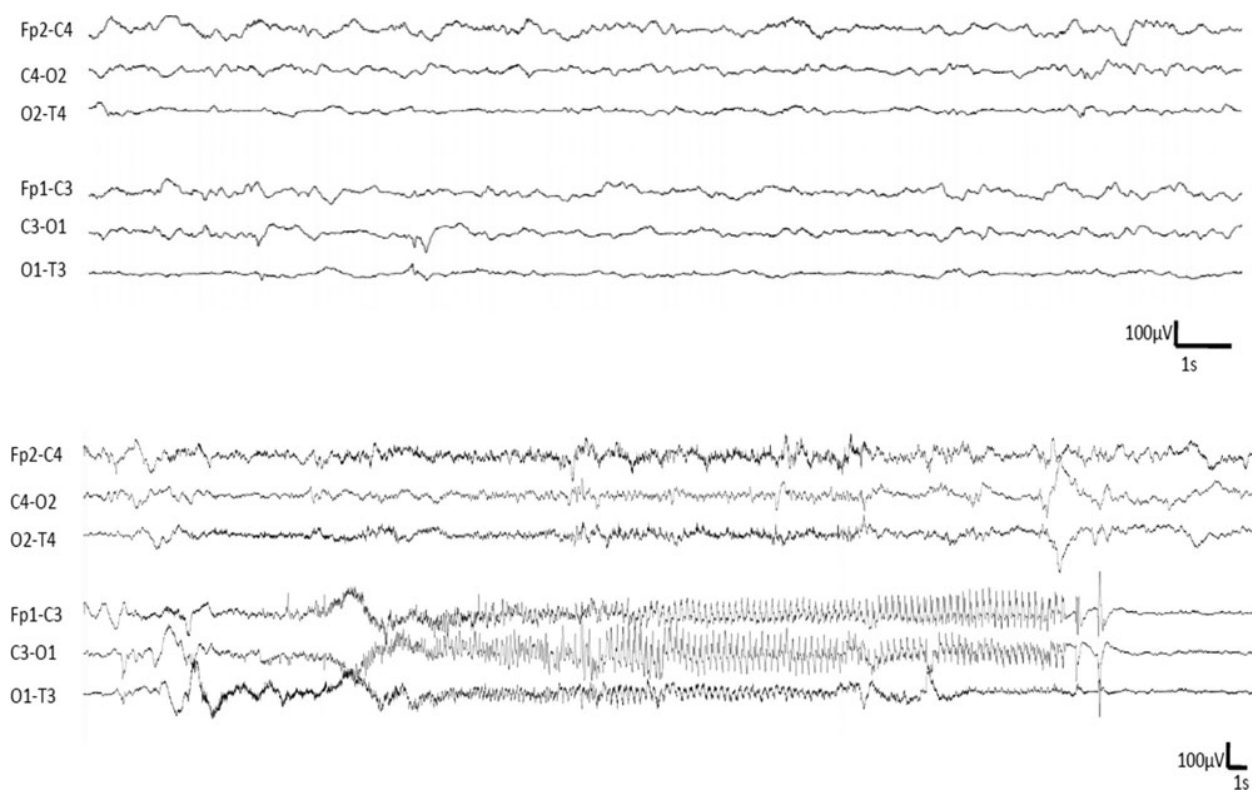

\section{Figure 2.}

Representative interictal (Top) and ictal EEG of a patient from the group 3 (Table I, patient I, I month of age), longitudinal montage. Interictal EEG shows multifocal spikes on a globally well-organized EEG. Ictal EEG shows a focal seizure originating from the anterior part of the left hemisphere. During the seizure, the patient has tonic asymmetric posture with clonic jerks of the right arm and head deviation. Focal seizures originating from the right hemisphere were also recorded for this patient. 
Group 1: EIEE with a suppression-burst pattern on the EEG leading to the diagnosis of Ohtahara syndrome $(\mathrm{n}=11)$, and improvement of all the parameters of epilepsy (seizure frequency and interictal EEG) within the first 2 years of life. Possible, but inconstant history of West syndrome during the course of epilepsy ( $\mathrm{n}=6$ of 11).

Group 2: Early onset epilepsy with focal or multifocal features and none evolved to West syndrome during the follow-up period $(\mathrm{n}=6)$.

Group 3: West syndrome with epileptic spasms and hypsarrhythmia, either after a period of focal/multifocal seizures, or other pattern $(n=5)$.

Among the two patients with an epilepsy beginning after 3 months, one patient was diagnosed "by chance" using array CGH to explore a case of intellectual disability with ataxic gait (patient 20). The second patient presented West syndrome as the first epileptic symptom in a context of initial psychomotor delay (patient 16).

\section{Developmental features}

At last evaluation, all patients had moderate to severe developmental delay. Patients with West syndrome underwent transient regression. Most patients had eye contact (92\%) and used this communication way in preference: only four patients used a few words to communicate (16\%). Seven patients $(29 \%)$ were able to walk and five of them had an ataxic gait. For patient 20, this ataxic gait was the reason for medical consultation. Patients 17, 22, and 24 could not walk but had ataxic standing.

\section{Other features}

Head circumference was normal from birth to the end of the follow-up period for all patients (Fig. 3). Axial motor tone was often low (Table 1), but axial hypertonia had been reported in two cases. Spasticity was never found in these patients.

Nonepileptic movements were described in $>54 \%$ : episodes of prolonged dystonic posture that could be mistaken for epileptic seizures were noticed in younger children; upper limb stereotypies, dyskinetic, and choreiform movements were also reported. Two thirds of cases older than 5 years of age had trembling posture of the head and trembling movements/prehension $(n=8)$. Trembling was the main nonepileptic movement disorder that was reported. Overall, nonepileptic movement disorders in older children became a major cause of concerns among parents, especially when epilepsy was controlled.

\section{Brain MRI of patients carrying a mutation of $S T X B P I$}

Brain MRI was performed in all STXBPI-mutated cases a few weeks after epilepsy onset (except for patient 20, who had MRI before epilepsy onset). Brain MRI did not show any obvious brain structural abnormality; however, patient 5 had an abnormal shape of the cortex in the right parietal area without any signal change. The brain MRI was repeated at least 1 year after in 17 cases. Results remained normal in $72 \%$. We observed progressive hypomyelination in three cases: patients 3 and 17 presented a frontoparietal hypomyelination, patient 18 had U-fiber hypomyelination. For patients 2, 16, and 22, diffuse cerebral atrophy was observed on a novel brain MRI at 1-, 15-, and 4-years-old, respectively.

\section{Relative frequency of STXBP1 mutations according to the epileptic features}

Among all the patients of our cohort that had a suppression-burst pattern on their first EEG $(n=44), 11$ were mutated for STXBP1 (25\%). The respective proportion of cases carrying a mutation of $S T X B P 1$ according to the epileptic syndrome was $25 \%$ in OS, $0 \%$ in early myoclonic epilepsy, $20 \%$ in nonspecific EOEE, and $4 \%$ in West syndrome as first epileptic symptoms (2/58). According to the age of onset, the rate of mutated patients was $11 \%$ in the group of early onset (before 3 months) and 3\% in the group of later onset.

\section{Discussion}

In this study, we described the major clinical features of STXBP1-related epileptic encephalopathy based on a large cohort of 284 patients. Twenty-two patients were carrying a mutation of $S T X B P 1$, and two patients were carrying a microdeletion that included STXBPI.

Our cohort does not reflect the relative proportion of each epileptic syndrome beginning within the first year of life, since the proportion of patients with an onset before 3 months is much higher than expected, and conversely the proportion of West syndrome is much lower. This is because our cohort was originally built to collect samples of patients with an early onset epileptic encephalopathy. We think that the respective rates of mutated patients are more relevant in the group of patients that began before 3 months than in the "older" group. Another study addressing specifically the question of the rate of mutated patients among epileptic syndrome beginning after 3 months of age would be useful to confirm that the mutation rate is much lower.

In our study, the initial EEG was abnormal in all patients. Suppression-burst and multifocal abnormalities were the most common patterns observed. When a suppression-burst was not found, EEG findings were still abnormal, showing multifocal paroxysmal abnormalities. This kind of abnormality is much less specific and was described in several other genetic EIEEs. However, these data allow distinguishing between STXBP1- and CDKL5-related epileptic encephalopathy, since in this later condition, interictal EEG is usually normal when the seizures begin. ${ }^{16}$

A major finding of our work is the head growth study: even with severe intellectual and motor disabilities, all 

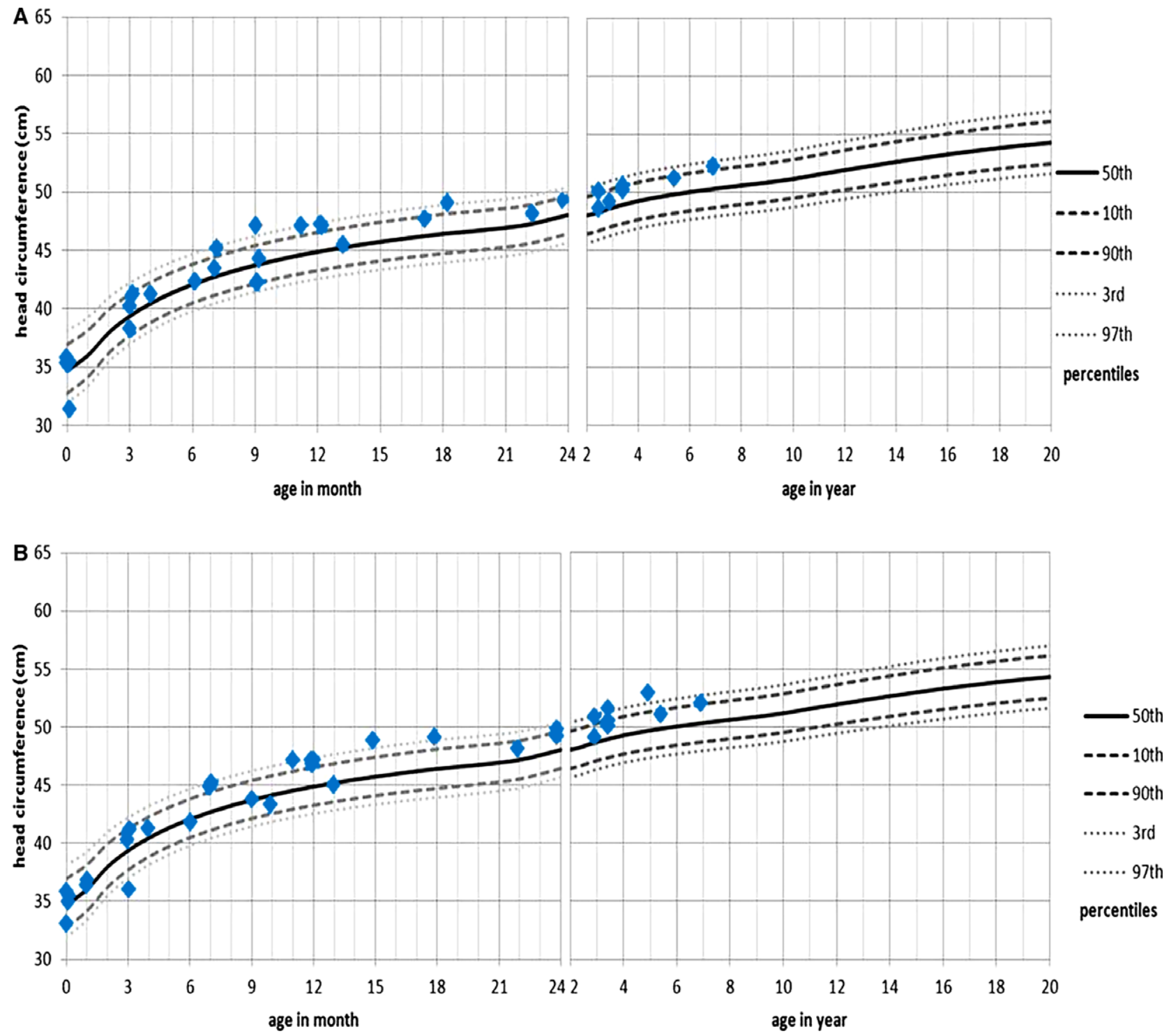

percentiles

Figure 3.

Evolution of patient's head circumference based from United States Head Circumference Growth Reference Charts. ${ }^{20}$ (A) Girls reference and (B) boys reference. Head circumference was normal for all patients.

Epilepsia (C) ILAE

patients had a normal head circumference from birth to the end of the follow-up. This parameter is easy to record, even for providers who are not epilepsy specialists. These data will allow the clinician not to indicate an $S T X B P 1$ screening in cases of epileptic encephalopathy with microcephaly, or intellectual disability with acquired or prenatal microcephaly.

More than half of the patients had nonepileptic movements. The most frequent were stereotypies, focused on the upper limbs, as described previously. ${ }^{8}$ Patients with a mutation in STXBP1 present severe developmental delay with limited autonomy in most cases. A small group of four patients seems to emerge, however: they display ambulatory ability with walking and possible communication by oral language. There seems to be no obvious correlation between this less severe phenotype and the type of mutation in STXBP1.

We did not find any brain structural or signal abnormality on the initial MRI, consistent with previous data from Saitsu et al. ${ }^{2}$ Brain MRI performed later in the course of the disease found progressive diffuse hypomyelination in 3 of 24 cases, and cerebral atrophy in 3 of 24 cases. These last three patients were not more severe than the others; two of them were able to walk.

The prospective follow-up allowed us to describe movement disorders that progressively became the major 
paroxysmal symptom in several patients: fluctuant and action-mediated trembling (reported in 11 of 24 cases), ataxic gait (reported in 9 of 24 cases), paroxysmal movements involving the face, neck, or extremities with stereotypical pattern (reported in 14 of 24 cases) often consistent with choreoballism as reported previously. $8,9,17$ These additional features helped us to make the diagnosis of STXBP1-related encephalopathy in at least one child with severe congenital ataxia, action-related tremor involving the head, normal head circumference, and normal brain MRI (patient 22).

More recently, de novo mutations of $K C N Q 2$ have been reported in EIEE5. ${ }^{18}$ The initial electroclinical presentation is similar to that of patients mutated in STXBP1, but seems to be more stereotyped: in our cohort, 16 patients carried a mutation of $K C N Q 2$, and for 15 of them, epilepsy started within the first week of life in a stormy way with asymmetric tonic or clonic seizures and discontinuous EEG. ${ }^{19}$ This was the case for only $50 \%$ of patients mutated in STXBPI. Thus the STXBP1-related epileptic encephalopathy seems to be more heterogeneous than $K C N Q 2$-related epileptic encephalopathy. Together, STXBP1 and KCNQ2 are mutated in more than half of patients with an Ohtahara Syndrome. On the other hand, an early onset epilepsy that is not associated with a discontinuous or suppression-burst pattern on the EEG, or that begins after the first week of life, is more probably in relation with a mutation in $S T X B P 1$ than with one in $K C N Q 2$.

We noticed a clear sensitivity to fever in three patients but none of them could, be diagnosis as Dravet syndrome, since they had developmental delay and abnormal interictal EEG from the beginning of the epilepsy.

Patient 20 had a clearly different phenotype: intellectual disability, congenital ataxia, and some epileptic seizures from adolescence. Of interest, the exonic deletion of STXBP1 has been identified "by chance," using array CGH. Patient 20 is in fact the only patient in the cohort not specifically addressed for an analysis of $S T X B P 1$. It is therefore very likely that this study underestimates the frequency of such phenotypes because the patients of our cohort were primarily sent for severe, early onset epilepsy.

In conclusion, we have shown that $S T X B P 1$ is mutated in approximately $10 \%$ of patient with an EOEE, and have found that they endure motor seizure, have an abnormal initial interictal EEG showing a suppression-burst pattern in half of the cases, an abnormal neurologic examination from the beginning of the disease, and a normal head circumference. Within the first year, half of them evolve to West syndrome. Subsequently, the epilepsy dramatically improves in the majority of cases, sometimes replaced by nonepileptic movements, and all of them have ongoing moderate to severe developmental delay with normal head growth. Altogether these features should lead the clinician to consider STXBP1 molecular screening.

\section{ACKnOWLedgments}

We would like to thank the patients' families and all the clinicians who sent DNA samples and clinical data on patients. We thank the Centre de Ressources Biologiques of La Timone Children's Hospital (Karine Bertaux, Cécile Mouradian, and Andrée Robaglia-Schlupp) for assistance with the samples used in this study. We thank Raphaelle Lamy for technical assistance. This work was funded by "Programme Hospitalier de Recherche Clinique" (PHRC IR 2011). MM was partially funded by INSERM "Contrat interface" and LV by Contrat Hospitalier de Recherche Translationnelle.

\section{Disclosure}

None of the authors has any conflict of interest to disclose. We confirm that we have read the Journal's position on issues involved in ethical publication and affirm that this report is consistent with those guidelines.

\section{REFERENCES}

1. Saitsu H, Kato M, Mizuguchi T, et al. De novo mutations in the gene encoding STXBP1 (MUNC18-1) cause early infantile epileptic encephalopathy. Nat Genet 2008;40:782-788.

2. Saitsu H, Kato M, Okada I, et al. STXBP1 mutations in early infantile epileptic encephalopathy with suppression-burst pattern. Epilepsia 2010;51:2397-2405.

3. Otsuka M, Oguni H, Liang J-S, et al. STXBP1 mutations cause not only Ohtahara syndrome but also West syndrome - result of Japanese cohort study. Epilepsia 2010;51:2449-2452.

4. Milh M, Villeneuve N, Chouchane M, et al. Epileptic and nonepileptic features in patients with early onset epileptic encephalopathy and STXBP1 mutations. Epilepsia 2011;52:1828-1834.

5. Weckhuysen S, Holmgren P, Hendrickx R, et al. Reduction of seizure frequency after epilepsy surgery in a patient with STXBP1 encephalopathy and clinical description of six novel mutation carriers. Epilepsia 2013;54:e74-e80.

6. Sampaio M, Rocha R, Biskup S, et al. Novel STXBP1 mutations in 2 patients with early infantile epileptic encephalopathy. J Child Neurol 2015;30:622-624.

7. Barcia G, Chemaly N, Gobin S, et al. Early epileptic encephalopathies associated with STXBP1 mutations: could we better delineate the phenotype? Eur J Med Genet 2014;57:15-20.

8. Kim YO, Korff CM, Villaluz MMG, et al. Head stereotypies in STXBP1 encephalopathy. Dev Med Child Neurol 2013;55:769-772.

9. Mignot C, Moutard M-L, Trouillard O, et al. STXBP1-related encephalopathy presenting as infantile spasms and generalized tremor in three patients. Epilepsia 2011;52:1820-1827.

10. Deprez L, Weckhuysen S, Holmgren P, et al. Clinical spectrum of early-onset epileptic encephalopathies associated with STXBP1 mutations. Neurology 2010;75:1159-1165.

11. Romaniello R, Zucca C, Tenderini E, et al. A novel mutation in STXBP1 gene in a child with epileptic encephalopathy and an atypical electroclinical pattern. J Child Neurol 2014;29:249-253.

12. Barcia G, Barnerias C, Rio M, et al. A novel mutation in STXBP1 causing epileptic encephalopathy (late onset infantile spasms) with partial respiratory chain complex IV deficiency. Eur J Med Genet 2013;56:683-685.

13. Hamdan FF, Gauthier J, Dobrzeniecka S, et al. Intellectual disability without epilepsy associated with STXBP1 disruption. Eur J Hum Genet 2011;19:607-609.

14. Carvill GL, Weckhuysen S, McMahon JM, et al. GABRA1 and STXBP1: novel genetic causes of Dravet syndrome. Neurology 2014;82:1245-1253.

15. Jette N, Beghi E, Hesdorffer D, et al. ICD coding for epilepsy: past, present, and future-a report by the International League Against Epilepsy Task Force on ICD codes in epilepsy. Epilepsia 2015;56:348355.

16. Bahi-Buisson N, Kaminska A, Boddaert N, et al. The three stages of epilepsy in patients with CDKL5 mutations. Epilepsia 2008;49:10271037. 
17. Kanazawa K, Kumada S, Kato M, et al. Choreo-ballistic movements in a case carrying a missense mutation in syntaxin binding protein 1 gene. Mov Disord 2010;25:2265-2267.

18. Weckhuysen S, Mandelstam S, Suls A, et al. KCNQ2 encephalopathy: emerging phenotype of a neonatal epileptic encephalopathy. Ann Neurol 2012;71:15-25.

19. Milh M, Boutry-Kryza N, Sutera-Sardo J, et al. Similar early characteristics but variable neurological outcome of patients with a de novo mutation of KCNQ2. Orphanet J Rare Dis 2013;8:80.

20. Rollins JD, Collins JS, Holden KR. United States head circumference growth reference charts: birth to 21 years. J Pediatr 2010;156:907913, 913.e1-2. 